\title{
Modeling Opportunistic Data Delivery in Dynamic Wireless Networks
}

\author{
Mary R. Schurgot ${ }^{1}$ \\ ECE Department \\ Stevens Institute of Technology \\ Hoboken, New Jersey USA \\ Email: mschurgo@stevens.edu
}

\author{
Cristina Comaniciu \\ ECE Department \\ Stevens Institute of Technology \\ Hoboken, New Jersey USA \\ Email: ccomanic@stevens.edu
}

\author{
Katia Jaffrès-Runser \\ University of Toulouse \\ IRIT-INPT ENSEEIHT, \\ Toulouse, France \\ Email: katia.jaffres-runser@irit.fr
}

\begin{abstract}
In this work we examine data delivery in dynamic wireless ad hoc networks that exchange packets opportunistically. Legacy mobile ad hoc network (MANET) protocols send packets along a predetermined path between a source and a destination. Node mobility, however, may create network partitions and a multi-hop link may not be present at the onset. Such node mobility may instead be leveraged to create an asynchronous path between a source and destination enabling opportunistic message exchange. In this work we propose an analytic model for this data exchange by defining a node's probability to forward packets to an encountered node. Drawing on results from mobility studies, we derive the statistics on the packet exchange and packet delivery performance for opportunistic networks. This model is validated for several simulation scenarios. The work provides a foundation for a one-to-one performance comparison of existing opportunistic forwarding protocols.
\end{abstract}

\section{INTRODUCTION}

Dynamic wireless ad hoc networks are characterized by intermittent connectivity and pairwise contact enabling opportunistic communication. In these ad hoc networks an endto-end path does not exist at any given time, but rather links between nodes change dynamically with varying network conditions, usually based on the mobility in the network. Additional terminology used to describe such networks includes delay tolerant networks, disruption tolerant networks, intermittently connected ad hoc networks, and opportunistic networks [1].

Message delivery in these networks is enabled by the storecarry-forward routing paradigm. Nodes leverage mobility to construct an asynchronous end-to-end path between a source and destination [2]. When a relay node carrying a message comes within transmission range of another node, the two nodes are said to be in contact. The decision to be made at this time is whether to forward the message to the encountered node or replicate the message by continuing to store the message and transferring a copy to the encountered node. Opportunistic routing protocols employ algorithms which decide to whom to forward and how much to replicate. These routing approaches range from naive flooding to networkaware predictive forwarding.

\footnotetext{
${ }^{1}$ The first author is now with LGS Bell Labs Innovations, Florham Park, NJ, USA. Email: maschurg@lgsinnovations.com.
}

Flooding protocols operate with the obvious tradeoff that the more you flood the better the chance to reach the destination and in a shorter time, but in turn you waste network resources, in terms of bandwidth, energy and storage. The design of most of the flooding based protocols does not account for the secondary effects of contention for the limited buffer space and the limited network capacity of typical wireless networks, thus resulting in message dropping and retransmissions, with a detrimental effect on performance [3] [4].

In contrast, network-aware algorithms aim to predict suitable relays for a destination. Simply adapting to the network conditions to support any opportunistic communication is no longer sufficient; instead, efficient forwarding schemes have been designed to balance the overhead from redundant copies with successful delivery and minimal delay. A new class of opportunistic routing protocols considers the underlying social network of the operators carrying the wireless devices to aid in the identification of efficient carriers for specific destinations. Social-based routing is rooted in the observation that the social connections between users vary more slowly than the wireless links; thus they serve as a good predictor of future contact [5]. Another motivation for this type of design is to quantify the wireless network structure to understand the role of nodes in connecting the sparse network for data delivery [1].

As the amount of knowledge available to the protocol increases, network performance with respect to average delay and delivery ratio improves [6]. With perfect knowledge of connectivity- past, present, and future state informationan optimization problem can be formulated. Solutions for benchmarking opportunistic routing protocols propose various oracle type schemes (e.g. [7]), which assume knowledge of all encounters in time and space to construct minimum cost routes. The authors in [7] extend their analysis of message forwarding to the multiple copy case with replication [8]. However, awareness of the entire wireless ad hoc network at individual nodes is not realistic in practice, let alone knowledge of the future network state.

Our approach to understanding data delivery is to use the statistical properties of node mobility to model data exchange during pairwise contact. A link exists between two nodes with some probability. This probability can be determined from the proposed mobility models in the literature [9]. 
Our model draws on the results that the intercontact time between successive node encounters follows a power law distribution with exponential tail [10]. Karagiannis et al. note that the exponential tail is an important characteristic based on the time scale of opportunistic networks. This statistical characterization of mobility has also been proposed in [11] and [12] to characterize the network graph. The authors in [11] seek to define a social-based routing protocol, which is not a goal here. The authors in [12] address the problem of distributing a message to multiple destinations where nodes have full knowledge of the number of relays and destinations possessing a copy of the message. In this work, we address a similar problem as in [12] but we do not make full knowledge assumptions and consider only the case where a single copy of a packet is present in the network.

In this work, our goal is not to propose a new routing protocol for opportunistic communication. Instead, we present a model to capture the exchange of data between nodes forming a dynamic wireless ad hoc network. Quantifying this data exchange is a first step towards a one-to-one protocol performance comparison as the forwarding probabilities can be selected to implement the forwarding policies for any practical routing protocol for opportunistic networks. In future work, the proposed model can be leveraged to formulate an optimization framework to derive better forwarding strategies for routing in intermittently connected networks. The purpose of this paper is to introduce and assess our proposed analytic model for data delivery in opportunistically connected wireless networks. Model results are validated using OMNet++ [13] simulations. Model accuracy has been tested for several mobility scenarios for a 4 and 10-node network. Our simulation results show that relative error between the proposed analytical model and simulation is very low (i.e. $0.075+/-0.04 \%$ for the 10 -node network), thus validating our model of data flows.

The paper is organized as follows. The network model is presented in Section II. In Section III we derive our proposed analytic model for packet exchange. We present analysis and simulation results in Section IV prior to concluding in Section V.

\section{Network Model}

The network of interest is an intermittently connected mobile network characterized by pairwise contacts between nodes in transmission range. In this model, the network is composed of a set $V$ of $N$ mobile nodes, decomposed into source, destination and relay nodes. Messages are routed in this network from one source node $S \in V$ to one destination node $D \in V$ in a multi-hop fashion using a store-and-forward paradigm. Source and destination do not participate in data relaying. As nodes come into contact, they exchange data to enable message delivery following the protocol model defined hereafter. In this work, we assume as well that the wireless link between a pair of nodes in contact has an infinite capacity, meaning all packets to be forwarded from one node to the other one are perfectly and instantaneously delivered.

\section{A. Mobility model}

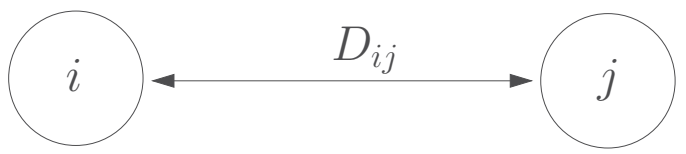

Fig. 1. The link between $i$ and $j$ is a function of the intercontact time $d$.

The opportunistic contacts between two nodes are commonly characterized using their intercontact time and their contact duration statistics. In this model, since we assume infinite transmission capacity when two nodes are in contact, we will not account for the contact duration in our model.

Intercontact time between two nodes $i$ and $j$ is characterized using a random variable $D_{i j}$ (cf. Fig 1) which is exponentially distributed with rate $1 / d_{i j}$ following the results of [10]. As such, average intercontact time of $i$ and $j$ is $d_{i j}>0$.

\section{B. Protocol model}

When a node $i$ encounters another node $j$, it has two options: do nothing or forward the entire contents of its buffer to $j$. As such, the forwarding decision of a node $i$ to node $j$ is a binary discrete random variable $F_{i j}$ that follows a Bernouilli distribution of probability $x_{i j}$. If $F_{i j}$ equals one, the content of the buffer of $i$ is forwarded to $j$.

In the rest of the paper, we will call $x_{i j}$ the forwarding probability between $i$ and $j$. This probability is conditional to the fact that $i$ and $j$ meet. In other words, if $j$ is the next node encountered by $i$ and $x_{i j} \neq 0$, node $i$ empties its buffer into the buffer of $j$ with probability $x_{i j}$. Note that if $x_{j i} \neq 0$, node $j$ empties its buffer into the buffer of $i$ with probability $x_{j i}$ during the same encounter.

We introduce the following terminology:

- A positive encounter for node $i$ is an encounter where it receives packets from another node. It increases the number of packets stored in its buffer. An encounter is positive for node $i$ if $x_{j i} \neq 0$.

- A negative encounter for node $i$ is an encounter where it forwards packets to another node. It decreases the number of packets stored in its buffer. An encounter is negative for node $i$ if $x_{i j} \neq 0$.

Note that an encounter can be both positive and negative.

\section{Node model}

Nodes can buffer a maximum of $K$ packets. We assume in the rest of this paper that all packets have the same size which is normalized to one.

To characterize the flows of packets transiting through node $i$, we define the following random variables:

$Q_{i}^{i n}$ the number of packets received by node $i$ at its next positive encounter,

$Q_{i}^{\text {out }}$ the number of packets forwarded by node $i$ at its next negative encounter,

$Q_{i} \quad$ the number of packets in the buffer of node $i$. 


\begin{tabular}{|c|c|c|}
\hline Parameter & Symbol & Definition \\
\hline source packet rate & $R_{s}$ & rate source $S$ generates packets \\
\hline intercontact time & $d_{i j}$ & average time between $i$ and $j$ meeting \\
\hline probability of contact & $P_{i j}$ & probability $i$ meets next node $j$ \\
\hline minimum time between encounter & $\Delta_{i}$ & average time until next encounter of node $i$ \\
\hline
\end{tabular}

TABLE I

NETWORK CHARACTERISTICS

At the time of a negative encounter for relay $i$, all packets in the buffer are forwarded to the encountered node if its forwarding decision is successful. With this specific packet exchange protocol, we always have $Q_{i}$ that equals $Q_{i}^{\text {out }}$ at negative encounters. As such, the following model only has to characterize the random variables $Q_{i}^{\text {out }}$ for all $N-2$ relay nodes (and not $Q_{i}$ ).

The destination node is not forwarding any packets. As such, only $Q_{D}^{i n}$ is of interest here. We assume that the source node is constantly generating packets with a constant rate $R_{S}$ and is able to empty its buffer at the time of negative encounters. As such, $Q_{S}^{i n}$ represents the random variable of the number of packets generated and, similarly to relay nodes, $Q_{S}^{\text {out }}$ represents the number of packets forwarded by $S$ at its next negative encounter.

\section{ANALYTIC DTN MODEL}

The aim of this paper is to propose an analytic model to derive the expected values $\bar{Q}_{i}^{\text {out }}$ for all relays, $\bar{Q}_{S}^{\text {out }}$ and $\bar{Q}_{D}^{i n}$, as a function of the forwarding probabilities and the mobility of the nodes. As we will highlight in Section IV, forwarding probabilities can model different common DTN routing strategies. In future work, the proposed model will be considered as a building block for deriving optimal routing strategies in an optimization problem where forwarding probabilities are varied. This section introduces first some preliminary calculations and then presents our analytic DTN model.

\section{A. Preliminary calculations}

a) Average time until the next encounter of node $i$ : or the minimum time between encounters, is the minimum of a set of exponential random variables, which is also exponentially distributed with a rate equal to the sum of the rates for all $\frac{1}{d_{i j}}$. It follows that the average time until node i's next encounter is:

$$
\Delta_{i}=\frac{1}{\sum_{j \neq i} \frac{1}{d_{i j}}}
$$

b) Probability $P_{i j}$ that node $i$ meets node $j$ next: The probability that $j$ is the next node encountered by node $i$ can be calculated based on the properties of the exponential distribution. The probability that node $j$ is the one with the minimum time between encounters is equal to the rate between $i$ and $j$ divided by the sum of all the rates. $P_{i j}$ can thus be defined in terms of $\Delta_{i}$ :

$$
P_{i j}=\frac{\Delta_{i}}{d_{i j}}
$$

c) Average time until the next negative encounter of node $i$ : A negative encounter for node $i$ meeting $j$ arises when $x_{i j}$ is positive. As such, the number of forwarding trials from $i$ to $j$ is modeled as a geometric random variable with probability $x_{i j}$, supported on the set $\{1,2,3, \ldots\}$. The average number of encounters with $j$ node $i$ experiences before a negative encounter happens is $1 / x_{i j}$. Thus, if these two nodes have an intercontact time $d_{i j}$, the intercontact time between negative encounters is:

$$
s_{i j}=\frac{d_{i j}}{x_{i j}}, \quad \forall x_{i j}>0
$$

The intercontact times between negative encounters retains the properties of the exponential distribution, but is now adjusted with rate $\frac{1}{s_{i j}}$. The average time until node i's next negative encounter is:

$$
\Delta_{i}^{\text {out }}=\frac{1}{\sum_{j \neq\{i, S\}} \frac{1}{s_{i j}}}
$$

This value is infinite for nodes that never choose to forward packets. However, in this work, we assume a relay node will always forward to the destination. Thus, $\Delta_{i}^{\text {out }}$ exists for all $i$.

d) Average time until the next positive encounter of node $i$ : Similarly, a positive encounter occurs when $x_{j i}>0$, i.e. node $j$ 's forwarding decision to $i$ is positive and the intercontact time between positive encounters with $j$ is:

$$
u_{i j}=\frac{d_{i j}}{x_{j i}}, \quad \forall x_{j i}>0
$$

Thus, the minimum average time until the next positive encounter for node $i$ is:

$$
\Delta_{i}^{i n}=\frac{1}{\sum_{j \neq\{i, D\}} \frac{1}{u_{i j}}}
$$

This value is infinite for nodes that never receive packets from any other node. For nodes having $\sum_{j \neq\{i, D\}} x_{j i}>0, \Delta_{i}^{i n}$ exists.

e) Probability $P_{i j}^{i n}$ that node $i$ meets node $j$ in a positive encounter: The probability that node $j$ is the next node involved in a positive encounter with $i$ can be calculated as in Eq. (2).

$$
P_{i j}^{i n}=\frac{\Delta_{i}^{i n}}{u_{i j}}
$$

In the case where $\Delta_{i}^{i n}$ does not exist for a node $i, P_{i j}^{i n}$ is set to 0 for all $j$. 


\begin{tabular}{|c|c|c|}
\hline Parameter & Symbol & Definition \\
\hline forwarding probability & $x_{i j}$ & probability node i forwards a stored packet to $\mathrm{j}$ upon encounter \\
\hline minimum time between positive encounter & $\Delta_{i}^{i n}$ & average time until node $i$ receives data \\
\hline minimum time between negative encounter & $\Delta_{i}^{\text {out }}$ & average time until node $i$ forwards data \\
\hline probability of positive encounter & $P_{i j}^{i n}$ & probability the next positive encounter of $i$ occurs with node $j$ \\
\hline received packets flow & $\bar{Q}_{i}^{i n}$ & average number of incoming packets per positive encounter \\
\hline stored packets & $Q_{i}$ & average number of packets accumulated between negative encounters \\
\hline transferred packets flow & $Q_{i}^{\text {out }}$ & average number of packets sent during a negative encounter \\
\hline
\end{tabular}

TABLE II

MAIN PARAMETERS AND DEFINITIONS OF OUR PROPOSED ANALYTIC MODEL

\section{B. DTN model derivation}

In this paper, we provide a derivation for the infinite buffer case, where each node can store an unlimited number of packets. Future work will also address the finite buffer case.

1) Case of the source node: The source $S$ generates packets with a constant rate $R_{s}$. When $S$ is isolated (i.e. not in contact with any other node), it accumulates the generated packets in its buffer with rate $R_{s}$. As such, it stores on average $\bar{Q}_{S}$ packets between negative encounters:

$$
\bar{Q}_{S}=R_{s} \Delta_{S}^{\text {out }}
$$

with $\Delta_{S}^{\text {out }}$ the average time until the next negative encounter of node $\mathrm{S}$.

When $S$ comes in contact with another node $j$ in a negative encounter, it empties its buffer into $j$ 's buffer. At this point in time, $\bar{Q}_{S}^{\text {out }}$ is equal to $\bar{Q}_{S}$.

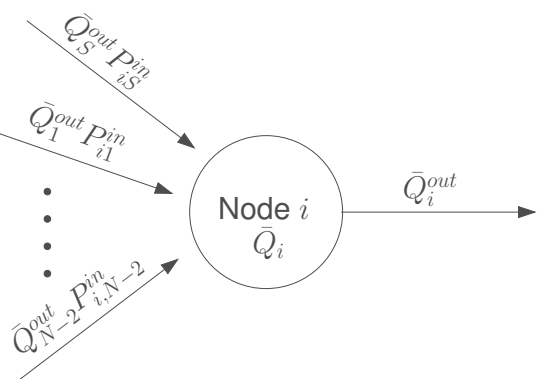

Fig. 2. The average flow of packets coming into node $i$ from $N-2$ relays and the source $S$ at positive encounters.

2) Relay node relationship: The average number of packets coming into node $i$ on positive encounters, $\bar{Q}_{i}^{i n}$, is the weighted sum of the average number of packets the other nodes of the network are forwarding on their negative encounters with $i$ (including the source node). This is illustrated in Fig. 2. In the following, the source has an index $S \in$ $\{1, . ., N-1\}$. For clarity, we extract $S$ from the subsequent summations. For node $i$, it has probability $P_{i j}^{i n}$ to receive packets from $j$ as stated in Eq. (7). Thus:

$$
\bar{Q}_{i}^{\text {in }}=\bar{Q}_{S}^{\text {out }} P_{i S}^{\text {in }}+\sum_{j \in[1, \ldots N], j \neq\{i, S, D\}} \bar{Q}_{j}^{\text {out }} P_{i j}^{i n}
$$

We can derive $\bar{Q}_{i}^{\text {out }}$, which is equal at the time of a negative encounter to the average number of packets accumulated in the buffer, as a function of $\bar{Q}_{i}^{i n}$. This relationship originates in the difference between the inter-arrival times of positive and negative encounters. To derive $\bar{Q}_{i}^{\text {out }}$, we can simply scale $\bar{Q}_{i}^{\text {in }}$ by the ratio $R_{i}$ of negative to positive encounters.

$$
\bar{Q}_{i}^{\text {out }}=\bar{Q}_{i}^{\text {in }} R_{i}
$$

with $R_{i}$ the ratio of the average time between negative encounters to the average time between positive encounters both defined in Eq. (4) and (6), respectively:

$$
R_{i}=\frac{\Delta_{i}^{\text {out }}}{\Delta_{i}^{i n}}
$$

Indeed, if more negative encounters than positive ones arise (i.e. $\Delta_{i}^{\text {out }}<\Delta_{i}^{i n}$ ), more packets are being forwarded to the rest of the network, and $\bar{Q}_{i}^{\text {out }}<\bar{Q}_{i}^{\text {in }}$. Similarly, if on average node $i$ experiences more often positive encounters than negative ones, it builds up packets in its buffer, and $\bar{Q}_{i}^{\text {out }}>\bar{Q}_{i}^{\text {in }}$. For the case of infinite time until the next positive encounter, i.e. when $\Delta_{i}^{i n}$ does not exist, $R_{i}$ equals 0 . Since $\Delta_{i}^{\text {out }}$ is never infinite, there is no undefined value of $R_{i}$.

Combining Eq. (9) and Eq. (10), we get $(N-2)$ equations:

$$
\frac{\bar{Q}_{i}^{\text {out }}}{R_{i}}=\bar{Q}_{S}^{\text {out }} P_{i S}^{\text {in }}+\sum_{j \in[1, . . N], j \neq\{i, S, D\}} \bar{Q}_{j}^{\text {out }} P_{i j}^{\text {in }}
$$

Note that for nodes with infinite time before a positive encounter $\left(R_{i}=0\right), Q_{i}^{i n}=0$ and computation of $Q_{i}^{\text {out }}$ is not relevant for this case.

3) Case of the destination node: The destination node always experiences positive encounters since it is an absorbing node. As such, $\forall i \in[1, . . N], i \neq\{D\}, x_{i D} \equiv 1 ; x_{D i} \equiv 0$. It follows that the average amount of packets received $\bar{Q}_{D}^{i n}$ per encounter is then:

$$
\bar{Q}_{D}^{\text {in }}=\bar{Q}_{S}^{\text {out }} P_{D S}+\sum_{i \in[1, . ., N], i \neq\{S, D\}} \bar{Q}_{i}^{\text {out }} P_{D i}
$$

4) Global network system: The unknowns of our model are $\bar{Q}_{S}^{\text {out }}, \bar{Q}_{D}^{\text {in }}$ and $\bar{Q}_{i}^{\text {out }}$ defined for all $i \in V, i \neq\{D, S\}$. $\bar{Q}_{S}^{\text {out }}$ is derived using the closed form expression of Eq. (8). Once Eq. (12) is expanded for each $i$, the linear system constructed by (12) and (13) is composed of as many equations 


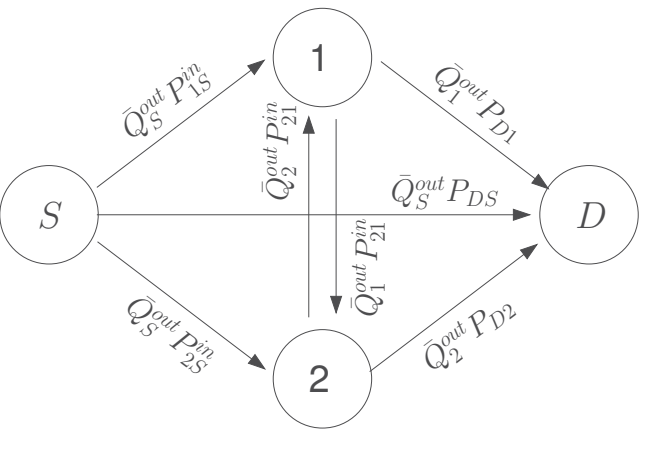

Fig. 3. Data flow in a 4-node network.

as unknowns. In vector form $A \cdot \bar{Q}=b$ :

$$
\begin{aligned}
& A=\left[\begin{array}{ccccc}
\frac{1}{R_{1}} & -P_{12}^{i n} & \ldots & -P_{1, N-2}^{i n} & 0 \\
-P_{21}^{i n} & \frac{1}{R_{2}} & \ldots & -P_{2, N-2}^{i n} & 0 \\
\vdots & \vdots & \ddots & \vdots & \vdots \\
-P_{N-2,1}^{i n} & -P_{N-2,2}^{i n} & \ldots & \frac{1}{R_{N-2}} & 0 \\
-P_{D 1} & \ldots & \ldots & -P_{D, N-2} & 1
\end{array}\right] \\
& \bar{Q}=\left[\begin{array}{c}
\bar{Q}_{1}^{\text {out }} \\
\bar{Q}_{2}^{\text {out }} \\
\vdots \\
\bar{Q}_{N-2}^{\text {out }} \\
\bar{Q}_{D}^{\text {in }}
\end{array}\right] \\
& b=\left[\begin{array}{c}
\bar{Q}_{S}^{\text {out }} P_{1 S}^{\text {in }} \\
\bar{Q}_{S}^{\text {out }} P_{2 S}^{i n} \\
\vdots \\
\bar{Q}_{S}^{\text {out }} P_{N-2, S}^{\text {in }} \\
\bar{Q}_{S}^{\text {out }} P_{D S}
\end{array}\right]
\end{aligned}
$$

Deriving the values for the average number of packets received at $D$ and forwarded by the relays requires us to solve the aforementioned $N$-by- $N$ linear system. The necessary condition is for the determinant of $A$ to be non-zero.

a) Illustrative example: For the four node network shown in Fig. 3 with a source $S$, destination $D$, and two relay nodes:

$$
\begin{aligned}
\frac{\bar{Q}_{1}^{\text {out }}}{R_{1}}-\bar{Q}_{2}^{\text {out }} P_{12}^{\text {in }} & =\bar{Q}_{S}^{\text {out }} P_{1 S}^{\text {in }} \\
-\bar{Q}_{1}^{\text {out }} P_{21}^{\text {in }}+\frac{\bar{Q}_{2}^{\text {out }}}{R_{2}} & =\bar{Q}_{S}^{\text {out }} P_{2 S}^{\text {in }} \\
\bar{Q}_{D}^{\text {in }}-\bar{Q}_{1}^{\text {out }} P_{D 1}-\bar{Q}_{2}^{\text {out }} P_{D 2} & =\bar{Q}_{S}^{\text {out }} P_{D S}
\end{aligned}
$$

This system can be solved if the determinant of $A$ follows:

$$
\frac{1}{R_{1}} * \frac{1}{R_{2}}-P_{21}^{i n} P_{12}^{i n} \neq 0
$$

Since $\forall(i, j) \in V \times V, d_{i j}>0$ and all nodes are forwarding data to the destination (i.e. $x_{i D} \equiv 1$ for $i \in V, i \neq D$ ), the determinant is always positive and can be solved.

\section{NumERICAL RESULTS}

\section{A. Simulations setup}

We use MATLAB R2011b to perform calculations and solve for $\bar{Q}_{S}^{\text {out }}, \bar{Q}_{D}^{\text {in }}$ and $\bar{Q}_{i}^{\text {out }}, \forall i \in V, i \neq\{S, D\}$. OMNeT++ IDE version 4.1 [13] is used as a framework to simulate the opportunistic network and validate our theoretical formulation. The simulation traces the number of packets buffered by each node. As in the previously defined model, packets cannot be discriminated and are transferred without knowing their content. As such, we do not model a forwarding rule only applying to packets that are new, i.e. different from the packets in the encountered node's buffer.

We create $\left(\begin{array}{l}n \\ 2\end{array}\right)$ self-messages [14] to indicate contact between a pair of nodes for all $n$ nodes in the network. The self-messages arrive according to an exponential distribution with rate $\frac{1}{d_{i j}}$. Communication is possible between a node $i$ and $j$ when a self-message selfMessage $\mathbf{~}_{\mathbf{i j}}$ arrives indicating pairwise contact. Upon the arrival of a self-message, node $i$ and $j$ decide with probability $x_{i j}$ and $x_{j i}$ respectively to transfer the entire content of their buffer to their counterpart. The buffer counters of each node are updated accordingly. There is also a source self-message indicating the arrival of a packet every $R_{S}$ time units. The source's buffer is then increased by one.

The simulation stores for each node $i$ the total number of packets forwarded $N_{f}(i)$ and the total number of negative encounters $E_{n}(i)$. Model values of $\bar{Q}_{i}^{\text {out }}$ are compared to their empirical counterparts $N_{f}(i) / E_{n}(i)$. For the destination node, the number of received packets $N_{r}(D)$ and the number of positive encounters $E_{p}(D)$ is traced. $\bar{Q}_{D}^{i n}$ is compared to $N_{r}(D) / E_{p}(D)$.

\section{B. Four node network}

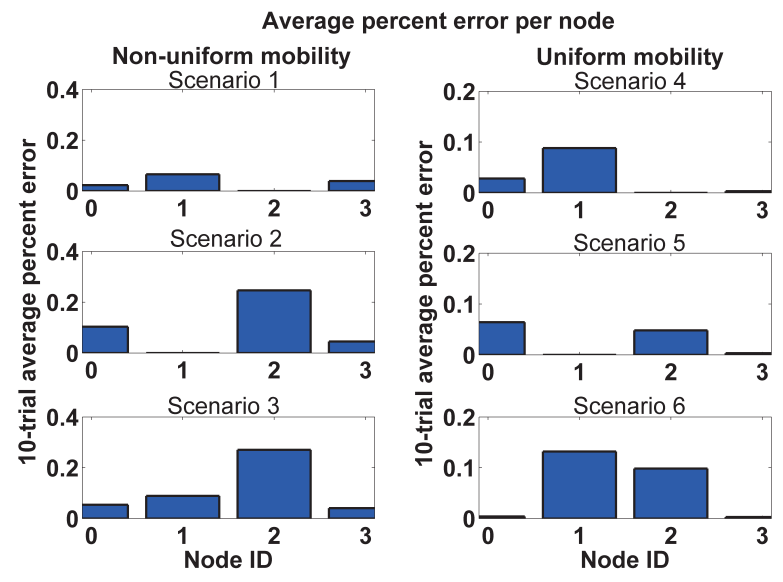

Fig. 4. Percent error results for a 4-node network. Node number 0 is the source and node number 3 is the destination.

Each of the first 6 scenarios is run for ten trials with different random seeds. The simulation is allowed to run for $10^{6}$ time units. The forwarding probabilities were modified for some example scenarios related to the four node network in Fig. 3. 
TABLE III

SIMULATION SCENARIOS AND ANALYTICAL RESULTS FOR AVERAGE INCOMING PACKETS FOR THE FOUR-NODE NETWORK

\begin{tabular}{|c|c|c|c|c|c|c|c|}
\hline \multicolumn{8}{|c|}{ Mobility scenarios } \\
\hline Scenarios S1-S3: & \multirow{2}{*}{\multicolumn{7}{|c|}{$\begin{array}{l}d_{01}=2 ; d_{02}=10 ; d_{03}=100 ; d_{12}=8 ; d_{13}=3 ; d_{23}=40 \\
d_{01}=d_{02}=d_{03}=d_{12}=d_{13}=d_{23}=10\end{array}$}} \\
\hline Scenarios S4-S6: & & & & & & & \\
\hline Forwarding Decisions & \multicolumn{7}{|c|}{ Analytic results } \\
\hline For all scenarios S1-S6: & & S1 & S2 & S3 & S4 & S5 & S6 \\
\hline$x_{i 0}=0, x_{3 i}=0, x_{i 3}=1$ for $i=\{1,2\}$ & $\bar{Q}_{S}^{\text {out }}$ & 1.961 & 9.091 & 3.226 & 5 & 5 & 5 \\
\hline For scenario S1, S4: $x_{01}=1, x_{02}=0$ & $\bar{Q}_{1}^{\text {out }}$ & 2.941 & 0 & 2.419 & 5 & 0 & 2.5 \\
\hline For scenario S2, S5: $x_{01}=0, x_{02}=1$ & $\bar{Q}_{2}^{\text {out }}$ & 0 & 36.364 & 6.452 & 0 & 5 & 2.5 \\
\hline For scenario S3, S6: $x_{01}=0.5, x_{02}=0.5$ & $Q_{D}^{i n}$ & 2.715 & 2.715 & 2.715 & 3.333 & 3.333 & 3.333 \\
\hline
\end{tabular}

Uniform and non-uniform mobility scenarios are considered with the source generating packets at a rate $R_{S}=1$ packets per time unit. In total, 8 different scenarios are presented for the 4-node network. Their properties are depicted in Table III.

For the first 6 scenarios, only 2-hop communications are allowed. More specifically, the two relay nodes 1 and 2 cannot exchange packets but only deliver the packets received from $S$ to $D$ (i.e. $x_{12}=x_{21}=0$ ). Depending on the values of $x_{S 1}$ and $x_{S 2}$, either 1 or 2 nodes are allowed by the source to carry packets to $D$. In practice, the forwarding decision of the source $x_{S i}$ may be set by external factors like information that $i$ is a good carrier for $D$ as used by the PRoPHET algorithm [3]; or $i$ is a highly central node as used in part by SimBetTS [15]; or perhaps $i$ and $D$ belong to the same community like the BUBBLE protocol considers [5].

Table III gives the analytical results for the four node network with average intercontact times shown. Here we use the term uniform mobility to refer to the scenarios in which the average intercontact time is the same for all node pairs and non-uniform mobility otherwise. The average percent error between analytical and empirical values for $\bar{Q}_{1}^{\text {out }}, \bar{Q}_{2}^{\text {out }}, \bar{Q}_{S}^{\text {out }}$ and $\bar{Q}_{D}^{i n}$ is plotted in Fig. 4 for each scenario. Percent error is very low for both non-uniform and uniform mobility scenarios. For uniform mobility, error is below $0.2 \%$ and for non-uniform mobility, error is below $0.4 \%$.

In scenarios 7 and 8, communication between nodes 1 and 2 is possible. Scenario 1 is modified in Scenario 7 with $x_{12}=0.5$ as node 1 empties its buffer into node 2 every two encounters. Thus, a 3-hop path from $S$ to 1 and then to 2 exists. In Scenario 8, the source forwards to node 1 and node 2 at every encounter while $x_{21}=0.5$. Fig. 5 displays the results for one trial of these three hop example scenarios. Error percent is still low for all nodes of the network.

\section{Ten node network}

Table IV extends the analysis to a ten node network. In this ten node network, $d_{i j}$ varies between 1 and 10 and is chosen randomly. Forwarding decisions are considered as binary: $x_{i j} \in\{0,1\}$ and chosen arbitrarily. $R_{S}$ is also changed to 10 packets per time unit. The simulation is run 5 times with 5 different seeds. As shown by these example scenarios, the described model provides a reliable framework for capturing data exchange in opportunistic wireless ad hoc networks.

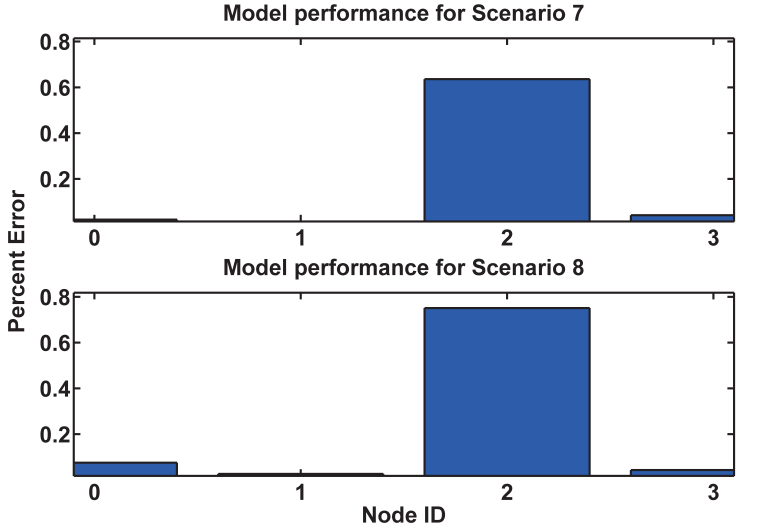

Fig. 5. Percent error results for a 4-node network using 3-hop long paths Node number 0 is the source and node number 3 is the destination.

Average error in percentage is below $0.169 \%$ for all nodes.

TABLE IV

RESULTS FOR A 10-NODE NETWORK

\begin{tabular}{lrrr}
\hline & Analytical & Simulation & $\%$ Error \\
\hline $\bar{Q}_{S}^{\text {out }}$ & 3.611 & 3.609 & 0.054 \\
$\bar{Q}_{1}^{\text {out }}$ & 2.045 & 2.045 & 0.002 \\
$\bar{Q}_{2}^{\text {out }}$ & 4.331 & 4.334 & 0.064 \\
$\bar{Q}_{3}^{\text {out }}$ & 3.932 & 3.929 & 0.061 \\
$\bar{Q}_{4}^{\text {out }}$ & 4.611 & 4.605 & 0.125 \\
$\bar{Q}_{5}^{\text {out }}$ & 9.178 & 9.174 & 0.050 \\
$\bar{Q}_{6}^{\text {out }}$ & 4.114 & 4.120 & 0.168 \\
$\bar{Q}_{7}^{\text {out }}$ & 6.673 & 6.664 & 0.132 \\
$\bar{Q}_{8}^{\text {out }}$ & 5.484 & 5.488 & 0.065 \\
$\bar{Q}_{D}^{\text {in }}$ & 4.738 & 4.736 & 0.028 \\
\hline
\end{tabular}

During our efforts to validate the model, we gained insight regarding the expected network performance. For the same mobility in scenarios 1-3 and scenarios 4-6, the same number of packets, 2.715 in the non-uniform case and 3.333 in the uniform case, are received at the destination, despite the variety of forwarding decisions in our example scenarios. With a change in forwarding probability, we show that the buffers of individual nodes accumulate more or less packets between negative encounters depending on the ratio $R_{i}$. Thus, the performance that is expected to vary is the average delivery delay as set by the mobility of the nodes. In future work, as 
we limit the capacity of relay buffers, we expect to see a dip in delivery. The current model allows us to identify those nodes that build up a large content store. For example, in scenario 2, the average number of packets leaving node 2 is much greater than that of node 1 when it is selected as the relay in scenario 1 .

\section{CONCLUSION}

In this work we have proposed a novel model for data packet forwarding in intermittently connected networks. A forwarding probability parameter for individual nodes was defined to capture forwarding policies of various practical routing protocols. Our analysis has determined the packet accumulation and data flow mechanisms from node to node with an impact on the computation of the expected final delivery of packets. Our analytical results were validated via simulations for some example networks. Future work will consider also the case of finite buffer capacity for individual nodes with an impact on determining the dropping probabilities at intermediate nodes. The proposed model provides a foundation for performance comparison of existing opportunistic forwarding protocols.

\section{REFERENCES}

[1] M. Schurgot, C. Comaniciu, and K. Jaffrès-Runser, "Beyond Traditional DTN Routing: Social Networks for Opportunistic Communication," IEEE Communications Magazine, vol. 50, no. 7, pp. 155-162, July 2012.

[2] C. Boldrini, M. Conti, and A. Passarella, "Exploiting users' social relations to forward data in opportunistic networks: The HiBOp solution,' Pervasive and Mobile Computing, vol. 4, no. 5, pp. 633-657, 2008.

[3] A. Lindgren, A. Doria, and O. Scheln, "Probabilistic Routing in Intermittently Connected Networks," in Service Assurance with Partial and Intermittent Resources, ser. Lecture Notes in Computer Science, P. Dini, P. Lorenz, and J. N. d. Souza, Eds. Springer Berlin / Heidelberg, 2004 vol. 3126, pp. 239-254

[4] Y.-C. Tseng, S.-Y. Ni, Y.-S. Chen, and J.-P. Sheu, "The Broadcast Storm Problem in a Mobile Ad Hoc Network," Wireless Networks, vol. 8, no. 2/3, pp. 153-167, Mar 2002.

[5] P. Hui, J. Crowcroft, and E. Yoneki, "BUBBLE Rap: Social-based Forwarding in Delay Tolerant Networks," IEEE Transactions on Mobile Computing, Dec. 2010.

[6] S. Jain, K. Fall, and R. Patra, "Routing in a Delay Tolerant Network," SIGCOMM Comput. Commun. Rev., vol. 34, pp. 145-158, Aug. 2004.

[7] T. Spyropoulos, K. Psounis, and C. Raghavendra, "Efficient Routing in Intermittently Connected Mobile Networks: The Single-Copy Case," IEEE/ACM Transactions on Networking, vol. 16, no. 1, pp. 63-76, Feb 2008.

[8] _ - "Efficient Routing in Intermittently Connected Mobile Networks: The Multiple-Copy Case," IEEE/ACM Transactions on Networking, vol. 16 , no. 1 , pp. $77-90$, Feb 2008

[9] D. Karamshuk, C. Boldrini, M. Conti, and A. Passarella, "Human Mobility Models for Opportunistic Networks," IEEE Communications Magazine, vol. 49, no. 12, pp. 157-165, Dec 2011.

[10] T. Karagiannis, J.-Y. L. Boudec, and M. Vojnović, "Power Law and Exponential Decay of Intercontact Times between Mobile Devices," IEEE Transactions on Mobile Computing, vol. 9, pp. 1377-1390, 2010.

[11] M. Wang and K. Nahrstedt, "Social Structure Based Routing of Intermittently Connected Network Using Contact Information," in Military Communications Conference, 2008. MILCOM 2008. IEEE, Nov. 2008, pp. 1-7.

[12] C. Singh, A. Kumar, R. Sundaresan, and E. Altman, "Optimal Forwarding in Delay Tolerant Networks with Multiple Destinations," in 201 International Symposium on Modeling and Optimization in Mobile, Ad Hoc and Wireless Networks (WiOpt), May 2011, pp. 228-235.

[13] "Omnet: Objective modular network testbed."

[14] A. Varga, "OMNeT++ User Manual, Omnet++ version 3.2." [Online]. Available: http://www.omnetpp.org/doc/omnetpp33/manual/usman.html
[15] E. Daly and M. Haahr, "Social Network Analysis for Information Flow in Disconnected Delay-Tolerant MANETs," IEEE Transactions on Mobile Computing, vol. 8, no. 5, pp. 606 -621, May 2009. 